\title{
The measurement of brain temperature. Influence of psychoactive drugs: a thermodynamic approach
}

\author{
Claude Marmasse ${ }^{1}$ \\ Institute of Psychiatric Research, Indiana University, \\ Medical Centre, Indianapolis, U.S.A.
}

EXTRAIT: Mesure de la température cérébrale. Influence des drogues psychoactifs: une approche thermodynamique. On a mis au point des méthodes très sensibles et très précises pour la mesure de la température d'une aire cérébrale déterminée; les bases physiques de la méthode et les détails des techniques neurochirurgicales utilisées sont décrites. Les composés psychoactifs provoquent en premier lieu une diminution de température. On peut également estimer par photométrie les variations d'énergie libre du processus. On discute les résultats en termes de réversibilité.

\section{INTRODUCTION}

There is now no doubt that a correlation between heat changes and neuronal activity exists (Aввотt, Hill \& How ARTH 1958). Furthermore, one class of changes in nervous activity was shown to be associated with changes in brain temperature: BUREs (1960) used thermocouples to measure temperature changes accompanying spreading depression. From a thermodynamical point of view, one would expect an initial drop in temperature following brain excitation. It must, however, be emphasized that as the organism is not a closed system, the unconsidered application of classical thermodynamics can be misleading.

The first object of the present work has therefore been to design sensitive, reliable and relatively rapid methods for measuring the temperature of the brain. In this respect, it must be emphasized that a temperature is in no case measured directly; what is measured is an equilibrium of temperature between two media, the medium whose temperature is measured and the sensing probe. In other words, the sensing unit will reflect the temperature of the surrounding medium only if the probe does not appreciably disturb the established thermic balance. If this is not the case, only the rate of cooling or of heating of the probe is measured. Although these latter techniques have been fruitfully used for the study of cerebral circulation, they are not within the scope of this paper. Unfortunately, the essential difference which exists between the true temperature of a tissue and the cooling rate of a transducer located in that tissue has not always

\footnotetext{
1 Present address: 9 quai de l'Abbé Grégoire, Blois, France.
} 
been recognized and confusions are frequent in the literature, as it is often difficult to know whether authors are referring to temperature measurements or to vasomotor phenomena.

\section{TECHNIQUES}

\section{Physical basis}

Bridge method. A thermistor used as temperature transducer can be placed as an arm in a Wheatstone bridge and the disequilibrium of the bridge recorded. In this procedure, there is generally a rather high density of current flowing through the thermistor; therefore, the bridge method was used in this investigation mainly for the study of different artifacts and routine studies.

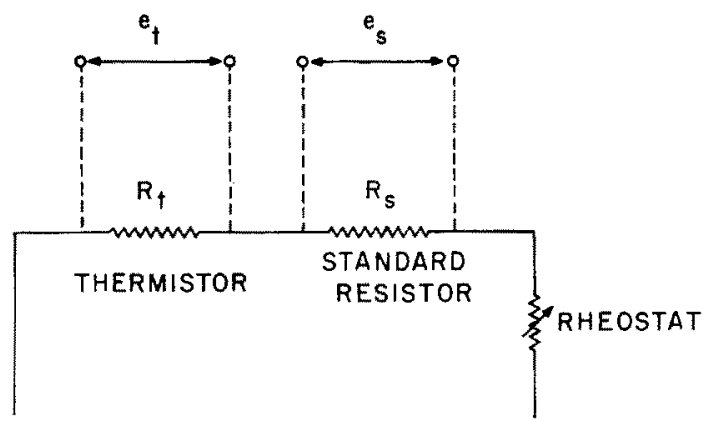

Fig. 1: Transducing circuit used for measurement of the temperature of the brain by the potentiometric method. Resistance $R_{t}$ of the thermistor is given by $R_{t}=R_{S} \frac{e_{t}}{e_{S}}$ where $e_{t}$ and $e_{S}$ are the differences of potential across $R_{t}$ and $R_{S}$ respectively

Potentiometric method. The thermistor is considered as a two-lead resistor. The transducing circuit is shown in Figure 1; it includes a series arrangement of a thermistor, a standard resistor and a rheostat used to vary the intensity of the current. The differences of potential across the thermistor and the standard resistor were measured by the opposition method. Conditions were generally chosen so that a constant current of $10.000 \mu \mathrm{A}$ flowed through the transducing circuit. Thus, the Joule effect which may induce a thermic excitation of the brain was kept to a negligible level (of the order of $10^{-4} \mu \mathrm{W}$ with a thermistor of low nominal resistance) and the computations were facilitated. Special techniques were used so that variations of $10^{-4} 0^{0} \mathrm{C}$ could be detected; they will be reported elsewhere.

\section{Construction of the transducing units}

The thermistors used were commercial thermistors of low nominal resistance (500 to 1000 ohms at $25^{\circ} \mathrm{C}$ ) and pearl-shaped (diameter: $0.17 \mathrm{~mm}$ ). They were mounted at 
the end of a thin polyethylene tube and coated with an insulating resin (INSL-ZE-33). Such a resin, the use of which is well known in electrophysiology, does not induce foreign body tissue reactions to any appreciable extent.

These transducers were used either fixed to a connector or as a probe. The connectors are shown in Figure 2. They were found to have a small and constant resistance

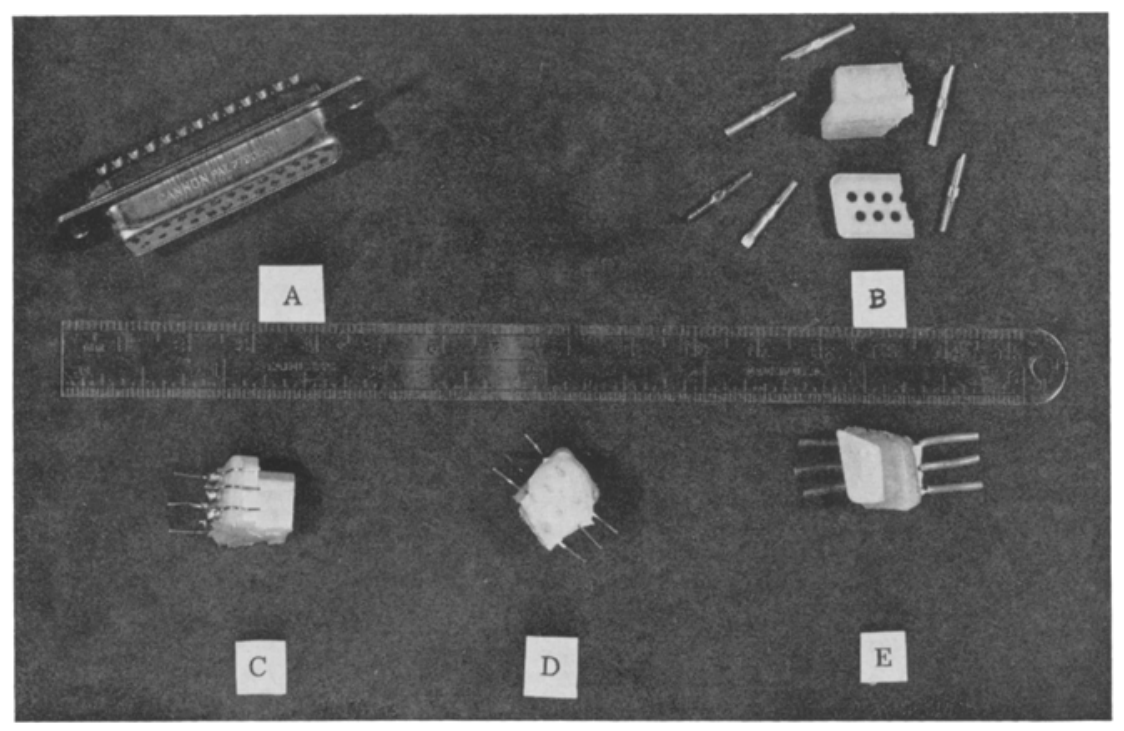

Fig. 2: Different steps in the construction of a connector used for the measurement of brain temperature. A: DB-25S Cannon connector; B: the connector disassembled and cut to appropriate size ( $1 \mathrm{~mm} ., 6$ outlets); C: appropriate portion reassembled with wires attached; D: coating of dental cement on base of connector; $\mathrm{E}$ : completed connector with protective tubing around wires and tape over outlets

of contact. Once the connectors were made, the top of the female part - fixed on to the skull at a later step - was covered with a piece of masking tape which is not pulled off before completion of the surgical procedure; thus the contacts are kept clean. These connectors were sterilized by exposure to germicide vapors.

\section{Surgical techniques}

The same techniques were used on either dogs or rabbits, although in the latter case the exiguity of the skull makes the operation more difficult. The animal is fixed on the operating table in the usual manner and the head, the neck and the shoulders carefully immobilized. The scalp is shaved, the ears fixed with adhesive tape so that the field is left free. Anaesthesia is obtained by infiltration with $2 \%$ Xylocaine (hydrochloride of diethylamino-acet-2,6-xylidide). After a longtitudinal incision, fields are placed and the superficial tissues dissected, as much as possible by blunt dissection. The skull is then exposed. 
In the case of a chronic preparation, the outer surface of the skull must be perfectly dry, so that the cement used (Caulk Dental Cement) will take. Bleeding, if it occurs, is controlled by means of high-frequency coagulation, bone wax and absorbable gelatine (Gelfoam).

Three or four little stainless steel screws are then affixed on the skull in such a way that they support the basis of the connector. When completed, new fields (red flags) are placed, and dental cement of a creamy consistency is applied. The smoothness and the regularity of the external surface of the dental cement block are very important; then the subsequent tissue irritation is kept to a minimum. The scalp is afterwards sutured with separate stitches, and an antibiotic (Bacitracin) applied locally.

Ten to twelve days later, the animal is generally ready for the second step, the implantation itself. The craniotomy is performed with a very small trephine, the dura incised with an ophtalmic knife or a well sharpened dissecting needle and the thermistor slipped into position. It is at this stage that it is possible - and indeed essential - to insure a good thermic contact between the thermistor and the brain area to be explored; this can be achieved by making use of the residual elasticity of the connecting wires so that the transducer is gently springloaded. The trepanation hole is obtured either with bone wax or, preferably, with a mixture of bone wax and bone scrapings (collected on the trephine). The manipulation is repeated as many times as necessary, depending on the number and the location of the transducers. The closure of the scalp is performed as previously described, but much time can be saved by using Michel clips.

In the case of acute preparations, the transducer is slipped through the trepanation hole, which is then closed with a little bone wax. Such transducers are best kept in position by anchoring them with silk or chromic catgut to small stainless steel screws fixed on the skull. The closure is effected as previously described but it is advisable to leave a solution of continuity on the scalp at the emergence point of the polyethylene tubing which encloses the connecting leads, in order to decrease the possibility of recording muscular artifacts (see section on artifacts).

In order to obtain satisfactory results, and especially in the case of non-chronic preparations, great care must be taken not to induce any appreciable swelling of the brain. The animal is best covered with an electric blanket and the absorbable gelatine used to control the bleeding moistened with warm saline; a reasonable speed and a good cooperation of the surgical team are also important factors for success.

\section{TEMPERATURE OF THE BRAIN}

\section{Artifacts}

Many artifacts can interfere with the accurate measurement of brain temperature, some due to the animal itself, others to the surgical procedure involved. We have studied the first class of artifacts on dogs which were subjected to experiments on the salivary reflex and the flexion of the hindleg within a Pavlovian framework. The dogs were implanted as previously described with connectors bearing two thermistors and 


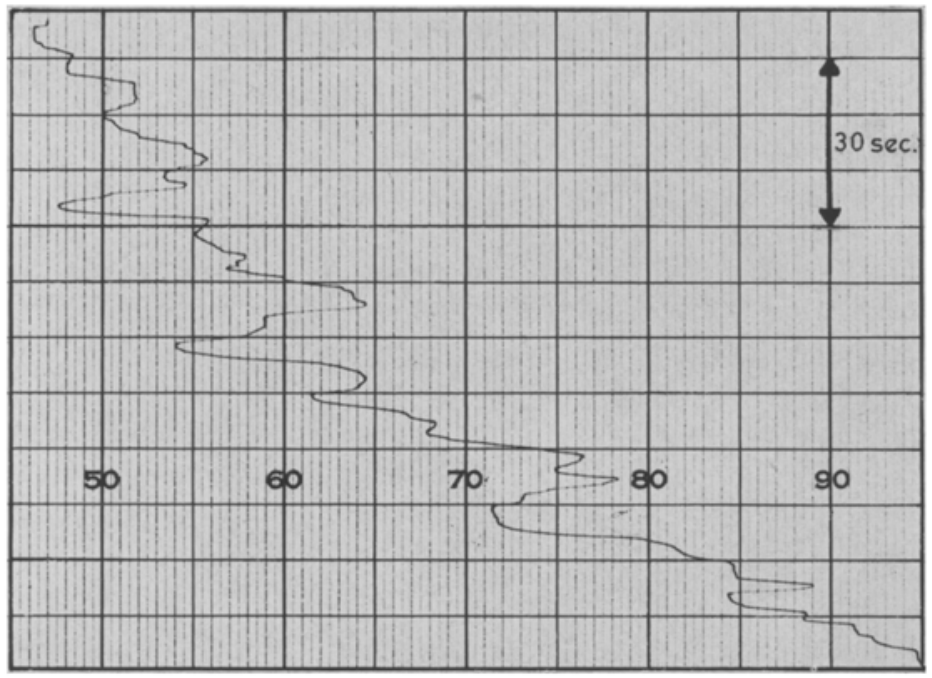

Fig. 3: Brushing of a dog; a minimally restrained situation. Very few spikes appear

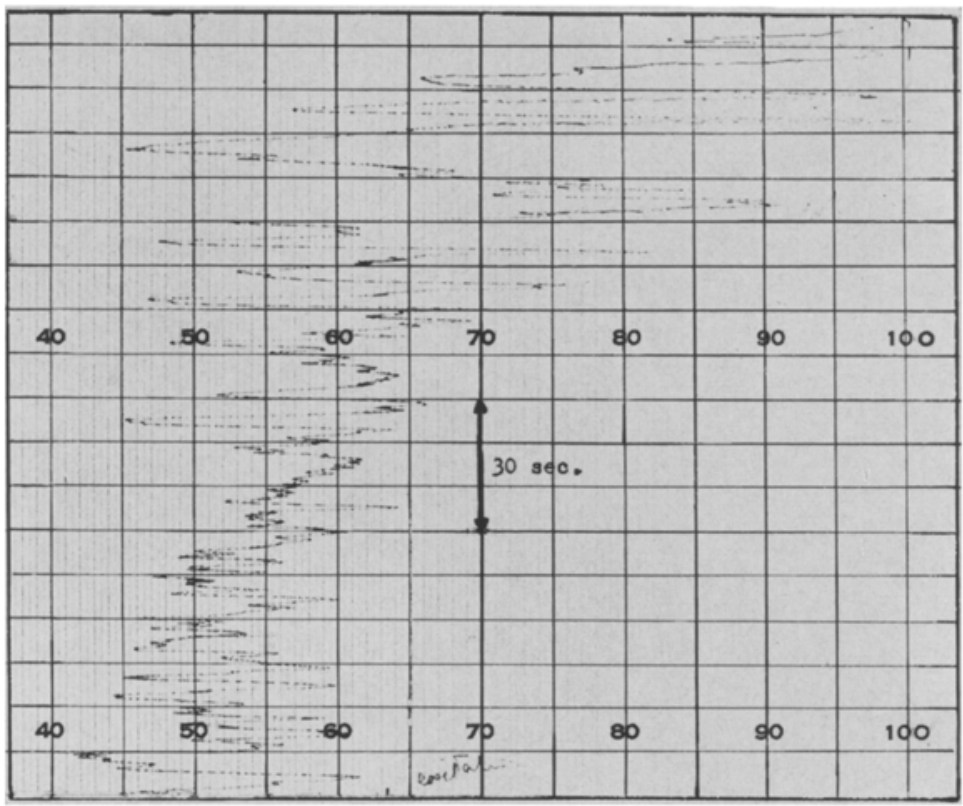

Fig. 4: Presentation of food to the same dog, placed this time in a typical Pavlovian conditioning apparatus. The dog is excited and shakes its head; many spikes appear 
two silver electrodes. The transducers were buried in soft tissues after the first surgical step.

After a recuperating period of approximately 10 days, the dogs were examined in order to ascertain continuity and insulation of the thermistors; several days later, the animals were studied again with the intention of obtaining baseline data at this stage. Each thermistors was in turn inserted as an arm of a Wheatstone bridge and the disequilibrium of the bridge continuously recorded with a potentiometric recorder. The current flowing through the thermistor was rather high, of the order of $1 \mathrm{~mA}$. Consequently, circulatory phenomena were monitored. Two different experimental situations were considered with the same dog; in the first, the animal was minimally restrained; in the second, the animal was restrained in a typical Pavlovian classical conditioning unit. This apparatus, when used with proper habituation procedures, has the advantage of reducing the movements of the animal.

When the dogs were quiet, rather smooth curves were obtained; but movements, and especially head movements were associated with sharp spikes. Figure 3 shows the type of record obtained when the animal is in a "free" situation and is gently brushed (this manipulation quietens the animal): few spikes are visible. Figure 4 is the reproduction of a record, obtained some days later, with the same animal in the restraining apparatus. In the sequence shown, the dog is given food, which induces very active head movements: the spikes, very sharp, and of a large amplitude, appear as bursts. Similar records were obtained with rabbits.

In the same way, with thermistors activated at a very low level (of the order of a few microamperes), muscular movements induce a shift of the difference of potential across the transducer. Spikes are therefore artifacts of muscular origin and must generally be discarded in the interpretation of temperature recordings.

The second class of artifacts is related to the surgical procedure itself; the phenomena are minimized when chronic preparations are used but are of major importance when considering non-chronics subjects. The immediate result of the neurosurgical procedure is to induce some swelling of the brain and often some thermic modifications in the tissues, mainly because of irritations, unavoidable bleeding and of vaso-motor effects provoked by the anaesthesia. It follows that it is necessary to wait, for a variable length of time depending on the reactions of the animal, after completion of the operation. Obviously the state of brain swelling can be checked by inspection and the temperature monitored. A greater source of difficulty, which is probably not always detected, at least during the course of the experiment, is the presence of a little blood at the tip of the transducer. Without considering the obvious physiological implications of such a situation, it must be emphasized that this liquid collection acts as a buffer, which can even, in certain cases, completely smooth out the temperature variations sought.

\section{Influence of psychoactive drugs}

Because of the many artifacts which might confuse the interpretation of the temperature records and in order to minimize the role of environmental variables, the 
experiments were performed in noiseless rooms with a subdued luminosity; in the same way, brusque movements of the experimenters were avoided as much as possible. That is to say that conditions were selected which concurred to a relaxed atmosphere.

It was quickly evident that rabbits were choice subjects, as they are not too nervous and tense and can be easily restrained in a way which creates a minimum of

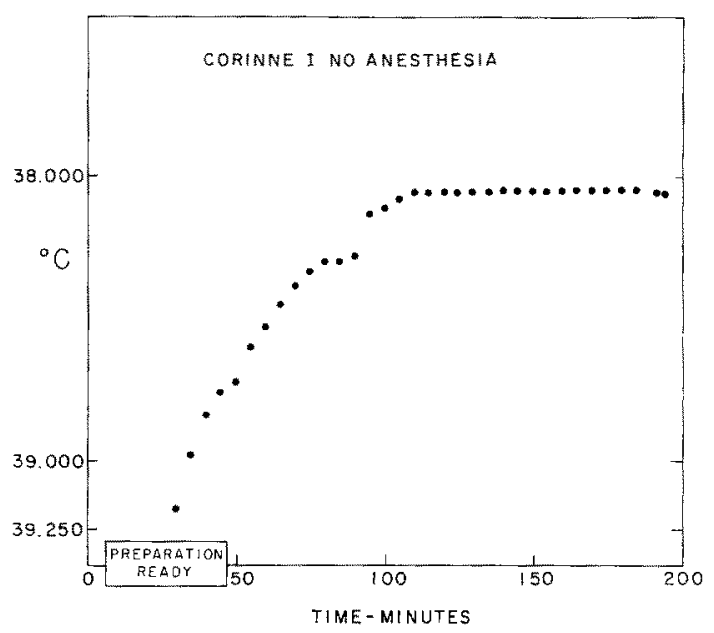

Fig. 5: Establishment of an equilibrium of the cortical temperature of a rabbit. Acute preparation; the time scale refers to the time elapsed since the beginning of the surgical procedure, which was completed in 30 minutes. The operation was performed with anaesthesia

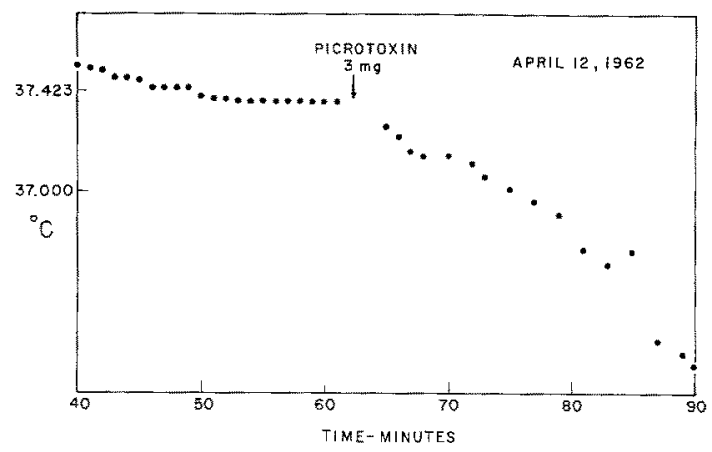

Fig. 6: Decrease of brain temperature of a rabbit by an injection of picrotoxin. The time scale refers to the time elapsed since the beginning of the surgical procedure which was completed in 40 minutes

discomfort to the animal. When all conditions were fulfilled, it was found that the brain temperature tended to level off; in the most favorable cases, the residual oscillations were generally amplified when the bridge method was used, probably because vasomotor phenomena were picked up by the transducers when, as mentioned above, a greater density of current was flowing through the thermistors. 
At times, a control injection of saline was made after the temperature had reached a plateau or had started to level off. When this manipulation was made in such a way that the animal was not excited, no change of the cerebral temperature was detected. On the other hand, if the animal felt the needle or was excited, a small drop in temperature was recorded (Fig. 8). In the same way, an environmental change

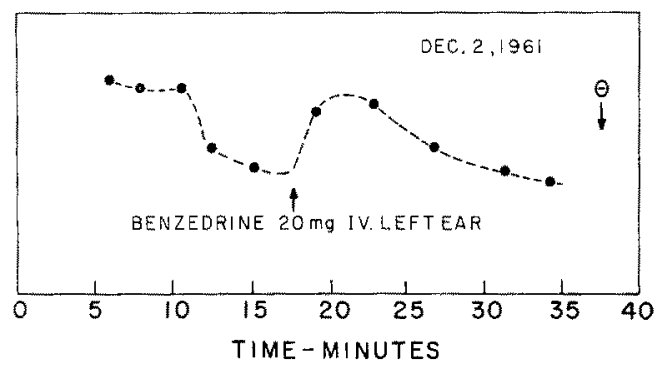

Fig. 7: Variation of brain temperature induced in a rabbit by an injection of benzedrine. The drug first provokes a drop in temperature. Acute preparation

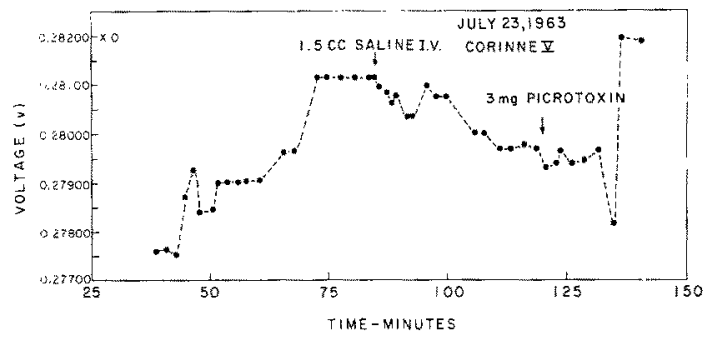

Fig. 8: Record of the brain temperature of a rabbit. Abcissa: time elapsed since beginning of surgical procedure; ordinate: voltage across thermistor. This figure shows an experiment especially difficult to interpret: although brain temperature levelled off, control injection of saline was followed by a small drop in temperature which might have been caused by fear. The brain then tended to read an equilibrium of temperature although oscillations of small applitude subsisted. An injection of picrotoxin induced, after a significant timelag, a definite decrease of temperature followed by a sharp increase (overshoot)

as slight as the displacement of any objects in the subject's visual field or a rapid change in the intensity of the light, induced a significant decrease in the brain temperature.

Some control experiments were performed. without anaesthesia, as it was reasoned that the influence of anaesthetics might have an action opposite to that of convulsive drugs on the temperature of the brain and because anaesthetics - be they local or general - always induce to some extent vasomotor changes. It is possible to make acute preparations, in the absence of anaesthesia, without causing major discomfort to a rabbit. In such a case, the cortical temperature levels off as can be seen in Figure 5 . The existence of such a plateau obviously offers many possibilities for the determination of the action of various compounds on the cerebral temperature.

The physiological and pathological state of the subjects is, as could be expected, 
a variable of importance. Although this aspect of the problem was not fully investigated, as only healthy animals belonging to an homogeneous group were used for most experiments, it was noticed on several occasions that the presence of fever was associated with cerebral thermic oscillations, the amplitude and frequence of which depending on the condition of a given animal.

The experiment described in Figure 6 unambiguously shows that picrotoxin induces a definite decrease in the cerebral temperature. Similar records were also obtained with other compounds, such as benzedrine sulfate, metrazol, etc. However,

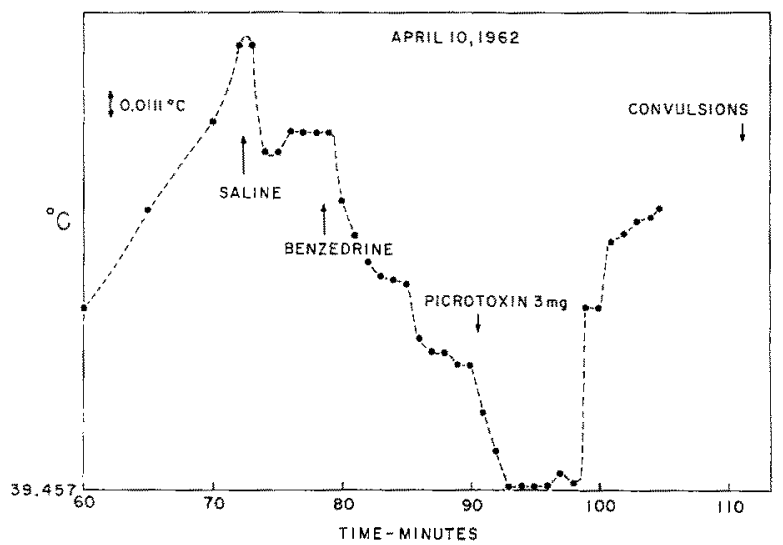

Fig. 9: Rabbit, acute preparation. Note principally the summation effect and the time-Iag between the drops in temperature induced by the convulsivant agents and the onset of the seizure

it must be emphasized that an experiment as "clearcut" as that in Figure 6 is an extremely rare occurrence. In many cases, the drop in temperature induced by psychoactive drugs lasts for a lesser time, as is shown in Figure 7.

In most cases, the thermic records obtained are very complex and their interpretation remains extremely difficult. Many different aspects can be noted: (1) Although the time-lag between the injection of the drug and its influence on the brain temperature is obviously smaller when the drug is injected in the marginal vein of the ear than when it is injected in the muscular masses of the hindleg, it can remain rather large, as in the case of Figure 8. (2) In many cases, the initial effect of a decrease in temperature is followed by an increase of temperature, which may last for a long time. It is this effect which was usually the only one observed by early experimenters because of the thermic inertia and of the relatively large heat capacity of most of the transducers used. The subsequent rise in temperature, when it exists, has sometimes the aspect of an overshoot, as depicted in Figure 8. (3) There may be a summation effect (Fig. 9). (4) There may be a marked delay between the change of temperature and the appearance of convulsions (Fig. 9).

The latter point may be of basic importance for an understanding of the mechanism of action of these psychoactive drugs. There seems to exist, at least for certain drugs, a correlation between a decrease of cerebral temperature and the onset of a 
seizure. More specifically, three rabbits, healthy and normal as far as could be ascertained, did not react at all to metrazol injections, even at the very high dosage of $45 \mathrm{mg} / \mathrm{kg}$ : no seizure could be detected by electrocorticographic and neurological examinations, nor any variation of temperature (monitored in different areas of the brain); while for all other subjects, a drop in temperature always preceded the onset of a seizure.

Under these circumstances, the variation in cerebral temperature is the manifestation of a primary phenomenon and shows that there is an essential action of psychoactive drugs on brain tissues before any apparent clinical manifestation.

Although much work remains to be done on this primary action, it can be stated that: (1) There is definite evidence of the existence of a threshold, and there is a large intraspecies variation to a given dose. For instance, an inconsistent response is obtained in rabbits with $0.75-1 \mathrm{mg} / \mathrm{kg}$ of benzedrine sulfate, but with $5 \mathrm{mg} / \mathrm{kg}$ a very clear response was obtained in most cases. In the same way, a decrease in brain temperature can sometimes be seen in rabbits with $0.05 \mathrm{mg} / \mathrm{kg}$ of picrotoxin; however, the phenomenon is generally best demonstrated with a dosage of $0.75 \mathrm{mg} / \mathrm{kg}$. (2) There is a large interspecies variation in response. (3) This action tends to be blocked by compounds like morphine or pentobarbital. (4) This primary action does not seem to be basically due to a vasomotor phenomenon because amphetamine, which causes a significant decrease in the cerebral temperature, has no marked or consistent effect on the cerebral circulation.

\section{THERMODYNAMIC APPROACH}

The variations in the cerebral temperature induced by psychoactive drugs are indicative of a variation of enthalpy. That a variation of free energy is associated with this process, can be deduced from experiments of FISCHER \& ZEMAN (1959). These authors showed that there is a relation between neuronal dye-absorption and nervous activity: nervous tissue excited by convulsivant agents or electrical current stains more intensely, all other conditions being equal, than tissue depressed, by nembutal for instance. An illustration of these facts will be found in Figures 10 and 11. (Due to the many photographic manipulations involved, these figures cannot pretend to reproduce the original difference in optical density of the preparations.) These experimental results were interpreted as being due to $\mathrm{pK}$ variations in certain proteins or as a reversible denaturation However, the fact that this difference in stainability was obtained in well-buffered systems is coherent with the hypothesis that the difference in the intensity of staining by the dyes used reflects a variation in the redox state of the system. It is more than plausible to consider that some electrons have been exchanged somewhere during the histochemical reaction. In these circumstances, if it is assumed that the dye uptake was quantitatively the same for excited and depressed tissues, the logarithm of the ratio of the intensity of staining is proportional to the variation of free energy involved in the process.

Accurate measurements of the difference in optical density of FISCHER \& ZEMAN's preparations are not possible at the present time. In the same way, the enthalpy vari- 


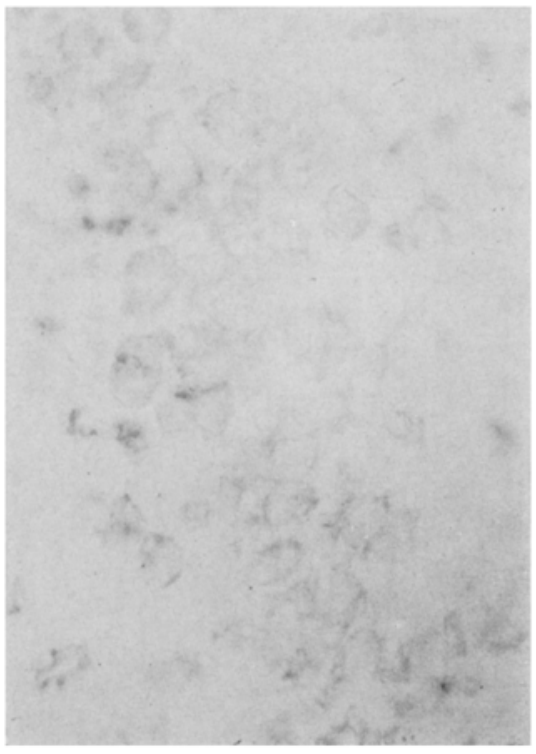

a

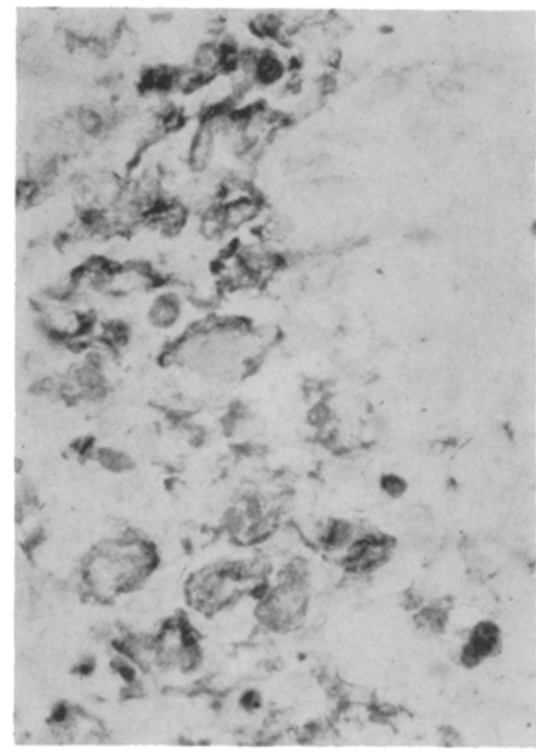

b

Fig. 10: Rat hippocampus. (a) after nembutal, (b) after picrotoxin. (Courtesy of Dr. R. Frscher)

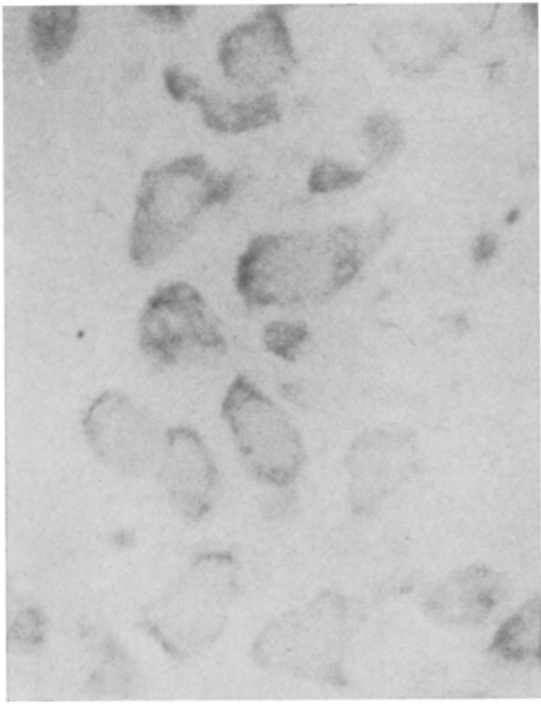

a

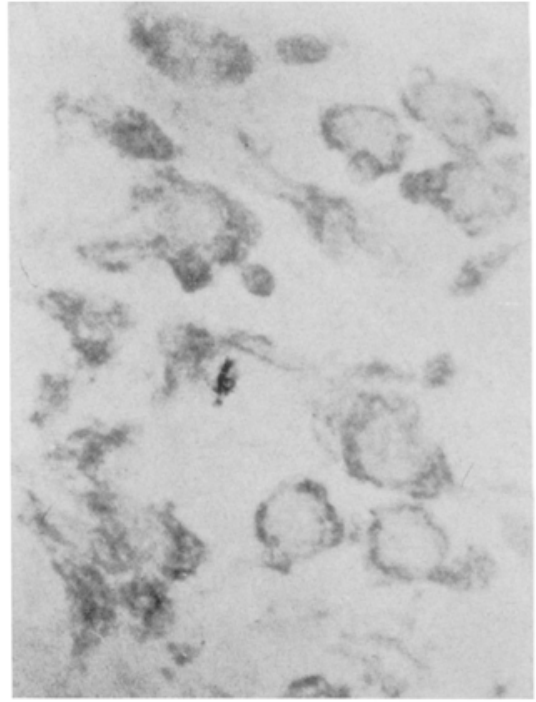

b

Fig. 11: Rat brain, pyramidal cells of hippocampus. (a) after nembutal anaesthesia, (b) after two electroconvulsive shocks. (Courtesy of Dr. R. FrSCHER)

ation can be estimated only roughly. It must be emphasized that, if the system is approximated for a short while by a closed system, it can be estimated that the brain tissue functions in conditions near reversibility. 
It is therefore apparent that the results of sophisticated techniques of cerebral thermometry, such as those developed in this laboratory, when used in conjunction with a new interpretation of FISCHER \& ZEMAN's findings, lead to a clearer view of certain basic cerebral mechanisms. The primary action of convulsivant agents is similar to that of physical and "psychic" stimuli. Thus, a direct study of entropic aspects of brain phenomena now becomes possible.

\section{SUMMARY}

1. Thermistors can be used to measure with high accuracy very small variations of temperature. In order to work in physiological conditions, the transducers were energized with very small currents and a potentiometric method of measurement was used.

2. Procedures were developed for the implantation of thermistors in cerebral areas.

3. Psychoactive drugs at first induce a decrease of cerebral temperature.

4. The corresponding variation of free energy of the process can be photometrically estimated.

5. The primary effect of convulsivant agents is similar to that of physical and "psychic" stimuli. A direct study of entropic aspects of brain phenomena now becomes possible.

\section{ACKNOWLEDGMENTS}

The experimental work described in this paper was carried out for the most part at the Institute for Psychiatric Research (Indiana University, Medical Center) of Indianapolis, with the technical assistance of Misses B. Brdalek, C. SPETH and Mr. J. SMall. The study of artifacts was carried out with the collaboration of Dr. A. RADELI at the Animal Conditioning Laboratory of the Department of Psychology, Indiana University in Bloomington. The help derived from stimulating discussions with Dr. W. ZEMAN (Department of Pathology, Indiana University) is gratefully adknowledged. Dr. R. FISCHER (Columbus Psychiatric Institute and Hospital, Ohio) kindly communicated to the author some of the photographs reproduced in this paper. The friendly help of Mr. F. BArLEY (Woods Hole, Mass.) for the scientific illustrations and Mr. B. Brayton (Falmouth, Mass.) for the photographs is also acknowledged. This paper was partly written while the author held a fellowship of the Commonwealth Fund.

\section{LITERATURE CITED}

Aввотt, B. C., Hill, A. V. \& Howarth, J. V., 1958. The positive and negative heat production associated with a nerve impulse. Proc. $R$. Soc. (B) 148, 149 ff.

Bures, J., 1960. Electrophysiology of the cerebral cortex. In: Electrophysiological methods in biological research. Ed. by J. Bures, N. Petrain \& J. Zachar. Czechoslovak Acad. Sci, Prag, Chapt. 8.

Fischer, R. \& ZeMan, W., 1959. Neuronal dye-sorption as a histochemical indicator of nervous activity, Nature, Lond. 183, 1337-1383. 


\section{Discussion following the paper by MARMASSE}

StRUbelt: (1) Haben Sie neben der Gehimtemperatur auch die Temperatur in anderen Organen gemessen? (2) Wie können Sie ausschließen, daß die beobachteten Änderungen der Gehirntemperatur durch Einwirkung der untersuchten Pharmaka auf den Gesamtstoffwechsel des Organismus verursacht werden?

Marmasse: (1) The answer is no. (2) It does not look likely at the present time that the primary action to which I was referring is due principally to a mechanism involving the general metabolism (see, for instance, the results of Fischer \& ZERMAN). However, even if this were the case, nothing in the thermodynamic approach described in this paper would be changed.

ZEISBERGER: (1) Where did you measure the brain temperature? (2) Did you also measure the blood flow through the brain? Changes in brain temperatures could also be explained by changes in the blood flow.

Marmasse: (1) The measurement of brain temperature was carried out in different areas of the brain. All areas of the brain investigated showed the same type of response, although the amplitude varied with the location. No complete mapping was made. (2) The influence of blood flow as the primary factor can be ruled out as it appears from the action of amphethamine: this compound consistently induces a drop in brain temperature while its action on cerebral circulation is not consistent or marked.

DeTchev: Kann nicht der Unterschied der Färbbarkeit durch die größere Absorbierbarkeit verursacht sein?

MARMASSE: It has never been proved that there is, in that case, a difference in dye capacity. It seems to me more plausible to consider that the capacity is the same in both cases and that the dye is fixed in different redox states. These are revealed by the difference in intensity which develops by reaction with the oxygen of the air. 